\title{
A Review and Comparison of FACTS Optimal Placement for Solving Transmission System Issues
}

\author{
A. Hernandez ${ }^{1}$, M.A. Rodriguez ${ }^{1}$, E. Torres ${ }^{2}$, P. Eguia $^{2}$ \\ ${ }^{1}$ Dept. of Power Grid Automation - Power Electronics \\ Ingeteam Power Technology - Technology \\ Parque Tecnológico de Bizkaia, Edif.108, 48170 Zamudio (Spain) \\ e-mail: aitor.hernandez@ingeteam.com, miguelangel.rodriguez@ingeteam.com \\ ${ }^{2}$ Department of Electrical Engineering \\ E.T.S.I.-Bilbao, University of the Basque Country (UPV/EHU) \\ Alameda Urquijo s/n, 48013 Bilbao (Spain) \\ e-mail: esther.torresi@ehu.es, pablo.eguia@ehu.es
}

\begin{abstract}
The current social impact of new transmission and distribution lines and the growth in environmental requirements has not lead to the expansion of the electric power grid but to the optimization of the existing assets. Flexible AC Transmissions Systems (FACTS), developed during the last decades of the past century, have become one of the most remarkable solutions for the optimization of the electrical power grid. Due to their flexibility, different types of FACTS have been proposed to solve similar transmission system operation problems. This paper reviews the FACTS devices used for solving these problems and the techniques used to optimize their location. The objective of the paper is to serve as a guide for selecting the right power system analysis and optimization technique for a given transmission system problem, and the most used FACTS for this purpose. The main operation issues that have been considered are: voltage control, assets optimization, line overloads and grid congestion, voltage stability problems, angle stability problems, contingencies and economic issues. In this study, the power system analysis and optimization methods have been divided into four main groups: classic optimization methods, technical criteria based methods, heuristic or meta-heuristic based methods and simulation based methods.
\end{abstract}

\section{Key words}

FACTS, power system analysis, optimization methods, FACTS location, transmission system problems.

\section{Introduction}

Nowadays, power systems are evolving from a relative static operation scenario to a more dynamic one due to the introduction of electricity markets, the high penetration of renewable and distributed generation and other drivers that introduce more variability and uncertainty in the operation of the power system. For example, under the electricity market operation, situations exist where the generation and consumption results coming from the market are limited by power transmission security and loadability constraints. In these circumstances, the Transmission System Operator (TSO) has two alternatives to solve this situation:

- De-meshing the power system. This is normally done by opening the busbar breaker of a substation next to the overloaded line/area or directly opening the overloaded line. This decision contributes to reduce the security level of the power system, increase transmission losses and reduce the lifetime of circuit breakers.

- Generation re-dispatching. In this case, some generation is replaced to avoid overloads while meeting demand requirements. This decision implies an economical compensation to the redispatched generator, an increment in the final electricity market price and, in case of renewable re-dispatches an extra negative environmental impact.

If the frequency of application of the previously mentioned solutions increases, a long term solution should instead be adopted. From the TSO's viewpoint there are three possibilities:

- Planning a new transmission line.

- Re-powering an existing line.

- Optimization of the existing power system by using FACTS and compensation devices.

Nowadays, environmental issues and social opposition to new transmission lines or to re-powering of existing ones make these solutions to be non-feasible for the expansion of the transmission grid. In this scenario, optimal use of the power system assets is one of the most important goals for TSOs. 
Flexible AC Transmissions Systems (FACTS) were firstly developed in the 1990's [1]. FACTS devices can help to alleviate transmission congestions but also other power system problems, which make this technology to be increasingly taken into account by TSOs. In addition, it can be said that this technology has reached maturity and that the cost of these power electronics based solutions has considerably decreased.

However, as the investment cost of FACTS is still high, their optimal location in the Power System is a crucial factor. Therefore, several FACTS location methods considering power system optimization techniques have been developed in the last years.

This paper presents the result of a bibliographic study about the different methods proposed in the literature to optimally select and locate FACTS devices. The study compares and classifies the methods according to different criteria, such as the type of FACTS considered, the specific purpose of the application of the FACTS device in the power system and the optimization technique applied in the methodology proposed. As a result, a crossed comparison of power system operation problems, optimization techniques and type of FACTS has been obtained, where the most suitable FACTS devices solutions are mentioned for each power system problem and the most accurate optimization methods are classified.

In the study, more than 180 references have been analyzed with the final objective of serving as a guide for selecting the right power system analysis and optimization technique for a given transmission system problem, and the most used FACTS for this purpose.

The paper is organized in 6 sections including this introduction. Section 2 presents the main transmission system problems analyzed in the study. Section 3 describes the power system optimization techniques used for optimal location of FACTS devices. Section 4 describes the different types of FACTS. Section 5 summarizes the main findings of the crossed comparison study and Section 6 contains the main conclusions.

\section{Transmission System Issues}

In the consulted references FACTS are applied to solve power system contingencies and other problems. In this section the different problems relieved by a FACTS solution have been classified in two groups: steady state and transient stability problems.

\section{A. Steady State Problems}

According to bibliography, FACTS based solutions can be applied with different purposes during steady state operation. In this paper, it is considered a steady state situation when the magnitude to control/optimize is not changing. The application of FACTS during steady state conditions has been classified for this study as proposed in [2]:

- Voltage control: Applications to optimize bus voltage values. These studies take into account the stability of power system voltages from the maximum and minimum admissible values.

- Transmission line overloads reduction: Applications to reduce the overload of a specific transmission line.

- Power system optimization: Applications of FACTS that deal, in general, with power system optimal power flow studies.

- Avoidance of generation re-dispatch and Power System Congestion: Applications that use FACTS to reduce congestions and redispatching of generators.

- Cost minimization: Applications with the objective of reducing the cost of the power transmission or the FACTS device cost optimization.

- Contingency analysis: Applications that deal with power system stability and security have been classified from the contingency analysis studies point of view.

\section{B. Transient Stability Problems}

FACTS are also applied to improve the performance of the power system under transient stability conditions. Transient stability considerations have been organized in two main groups, those related with voltage and angle stability, respectively.

- Voltage Stability and Voltage Collapse. The application is focused on the voltage stability of the system and the determination of the weakest bus of the power system and possible source of future transient stability problems [3].

- Angle Stability and Power Oscillations. In this case, the application purpose is to minimize and damp power oscillations.

\section{Power System Optimization Techniques}

There are several power system optimization techniques proposed in the literature that deal with the problem of optimal placement of FACTS devices. In this section, references are classified according to the most common techniques, with the purpose of determining which technique has been more frequently applied and so, which one is the most suitable for a given power system study dealing with FACTS optimal location. Analysis methods have been divided in 4 groups: classical optimization methods, technical criteria based methods, heuristic and meta-heuristic methods, and simulation based methods [4].

\section{A. Classical Optimization Methods}

These methods are based on mathematical equations which result in the solution of the system. The system optimization is obtained by the application of an iterative process that can be solved by Linear Programming (LP), Mixed-Integer Programming (MILP), Mixed-Integer Non-Linear Programming (MINLP), Non-Linear Programming (NLP) and Quadratic Programming (QP) [5]. 


\section{B. Technical Criteria Based Methods}

These methods use technical criteria derived from a particular sensitivity analysis, for steady state studies, and from a modal analysis when referring to dynamic and transient analysis.

Sensitivity based analysis are based on indexes, which can be defined depending on the variables under consideration. Thus, real power performance index, single contingency index, voltage stability L-index, etc., can be found in the literature.

\section{Heuristic and Meta-heuristic Methods}

Heuristic and meta-heuristic methods are computational methods that optimize a number of candidate solutions in an equation system by iteratively trying to improve the objective function.

References analyzed consider 18 heuristic and metaheuristic methods for the optimal location of FACTS: Evolution Strategy (ES), Evolutionary Programming (EP), Genetic Algorithm (GA), Simulated Annealing (SA), Tabu Search (TS), Ant Colony Optimization (ACO), Particle Swarm Optimization (PSO), Differential Evolution (DE), Harmony Search (HS), Bees Optimization (BO), Fuzzy Logic (FL), Trajectory Sensitivity Analysis (TSA), Dual Programing (DP), Gravitational Search Algorithm (GSA), Curve Space Optimization (CSO), Decomposition Coordination Method (DCM) and ELECTRE - III.

\section{Simulation Based Methods}

In some other references, simulation based methods have been used to figure out the most appropriate FACTS location. This group includes simulation tools such as: PSAT, Matlab, continuous power flow simulations and time domain simulation methods.

\section{Flexible AC Transmission Systems}

According to [6] a FACTS device can be defined as: "A power electronic based system and other static equipment that provide control of one or more AC transmission system parameters to enhance controllability and increase power transfer capability".

FACTS can be classified under multiple criteria: the application, technology, type of connection to the power system, installation cost per MVA, dynamic response of the FACTS and some other consideration.

In this study, 7 FACTS devices have been taken into account: Static Synchronous Compensator (STATCOM), Static Series Synchronous Compensator (SSSC), Unified Power Flow Controller (UPFC), Static Var Compensator (SVC), Thyristor Controlled Series Capacitor (TCSC), Thyristor Controlled Series Reactor (TCSR), Thyristor Controlled Phase Shift Transformer (TCPST) and Phase Shift Transformer (PST). These FACTS are the most mentioned or known FACTS, but other types with similar characteristics have been considered as well: Thyristor
Controlled Voltage Regulator (TCVR), Voltage Sag restorer Compensator (VSrC), Hybrid Power Flow Controller (HPFC), Optimal Unified Power Flow Controller (OUPFC) and Thyristor Controlled Phase Angle Regulator (TCPAR).

\section{Results}

In this analysis over 180 references have been evaluated, using [1]-[8] as a starting point for the study. Each reference has been classified according to the application problem, the proposed optimization method and the most appropriate FACTS device for this purpose. Fig. 1 shows the number of references classified according to the type of optimization method used.

In the analysis done, it has to be considered that in several references multiple transmission problems, FACTS, and optimization techniques are addressed.

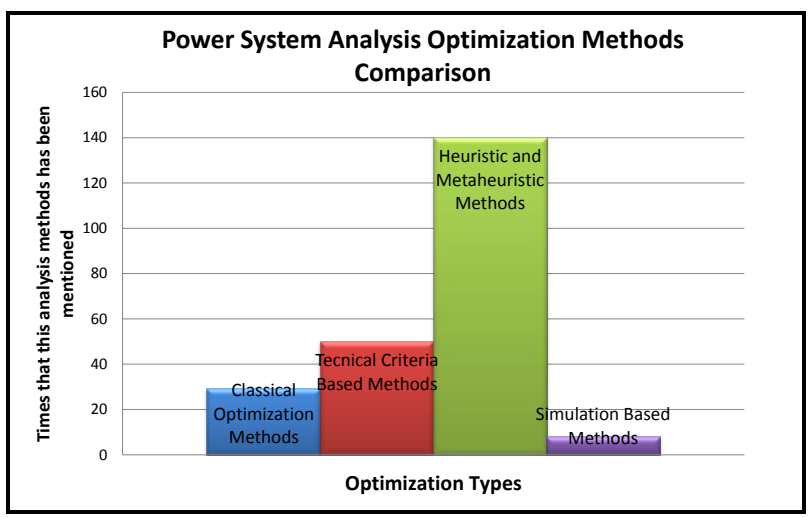

Fig. 1 Comparison of optimization methods by group

Thus, in [9]-[11], some examples of classical optimization methods are mentioned, where an optimal placement of a TCPST, TCSC and UPFC are studied. In references [12]-[15], some technical criteria based methods are mentioned for series and parallel connected SVC, SSSC, UPFC and TCSC. Some of the studied heuristic and meta-heuristic methods are described in [16]-[18], TCSC, TCPST, TCVR and UPFC are optimally placed based on GA, PSO, FL and DE methods. Then, in [19]-[22] simulation software has been used to locate FACTS. However, hybrid methods, based on a combination of some of the previous named methods, have been proposed in [23]-[25].

In Fig. 2 a comparison of all evaluated techniques is shown. The first 3 columns are the classical optimization methods NLP, LP and MINLP respectively. The columns 4 and 5 are Technical Criteria based techniques where 4 are index sensitivity based methods and 5 are modal analysis methods. From 6 to 23 all the analysed heuristic and meta-heuristic techniques are shown and finally, the last column corresponds to simulation based methods. 


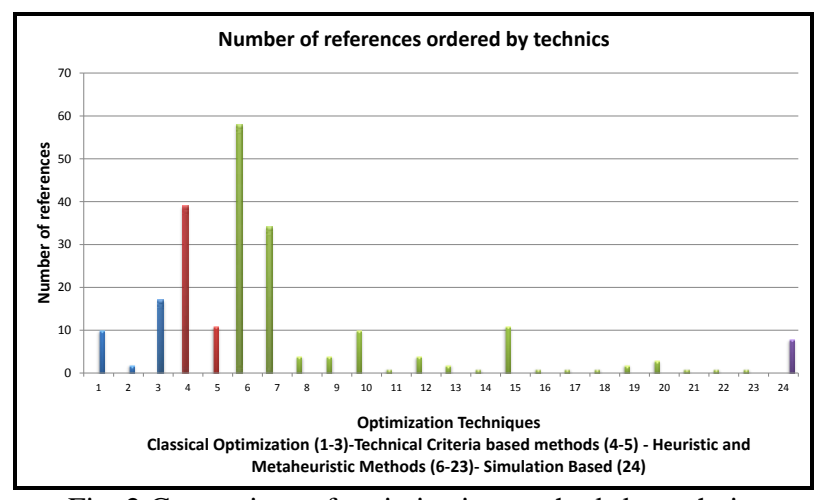

Fig. 2 Comparison of optimization methods by technique

The most used methods are based on meta-heuristic techniques, but they are widely distributed. In Fig. 2 it can be seen that the most used techniques are:

- GA (Heuristic or meta-heuristic methods).

- Index based sensitivity methods (Technical Criteria methods)

- $\quad$ PSO (Heuristic or meta-heuristic methods).

- MILP and MINLP (Classical Optimization methods).

- DE (Heuristic or meta-heuristic methods)

- EP (Heuristic or meta-heuristic methods).

Using the classification, a cross comparison has been done in order to determine which is the most suitable FACTS solution for each power system application/problem and which optimization method has been more deeply studied for the same mentioned application.

Fig. 3 shows the number of times that each FACTS device has been studied for a given transmission application/problem. It can be seen that the classical series and parallel FACTS devices, TCSC and SVC, are the most applied ones.

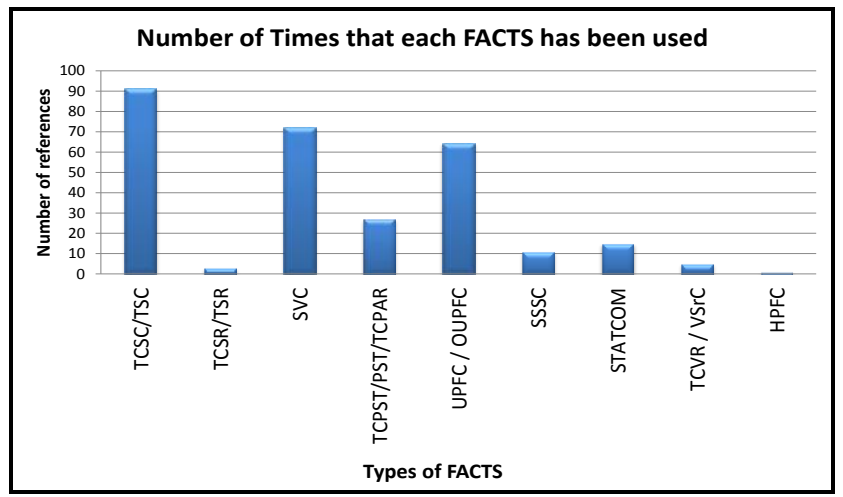

Fig. 3 Number of times each FACTS device is used

Most of the times the TCSC has been used for power flow control and optimization and for angle stability applications. The SVC has been used for voltage control and voltage stability. The UPFC is the 3rd more cited FACTS. This device has been used for multi-objective optimization applications due to its flexibility.

Regarding Voltage Source Converter (VSC) based FACTS, the SSSC and STATCOM have been introduced in the last decades. This explains why they are less mentioned than other types of FACTS.
Fig. 4 represents the number of times that FACTS devices have been considered as a solution to the different transmission system problems. As it is shown, the main objective for the FACTS optimal location has been the optimization of the power system assets. Generally, this issue has been studied in conjunction with cost optimization. The second more studied application has been the voltage control and the third one is the reduction of line and transformer contingency severity.

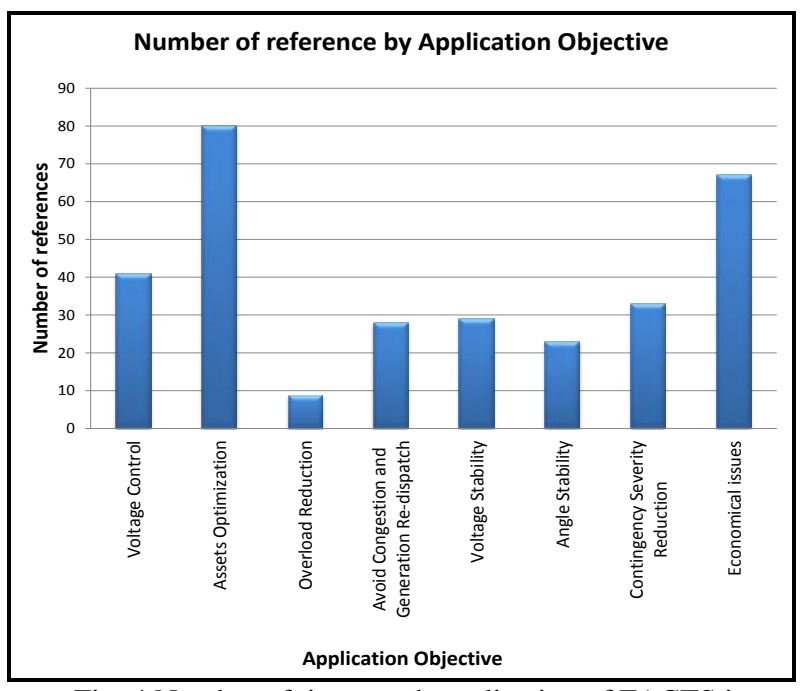

Fig. 4 Number of times each application of FACTS is referenced

In Fig. 5 a cross comparison of FACTS and their applications has been done, according to the number of papers where a type of FACTS has been studied. On the left, the applications are mentioned, from top to bottom: Voltage Control, Assets Optimization, Line Overload Limiting, Congestion and Re-dispatch Avoidance, Voltage stability and collapse, Angle stability, N-1 Contingency criteria fulfilment and Transmission cost minimization. On the right, the scale shows the number of times that a certain FACTS has been used. On the bottom, the different FACTS are mentioned like: TCSC/TSC, TCSR/TSR, SVC, TCPST/PST, UPFC, SSSC, STATCOM, TCVR/VSrC and HPFC, respectively.

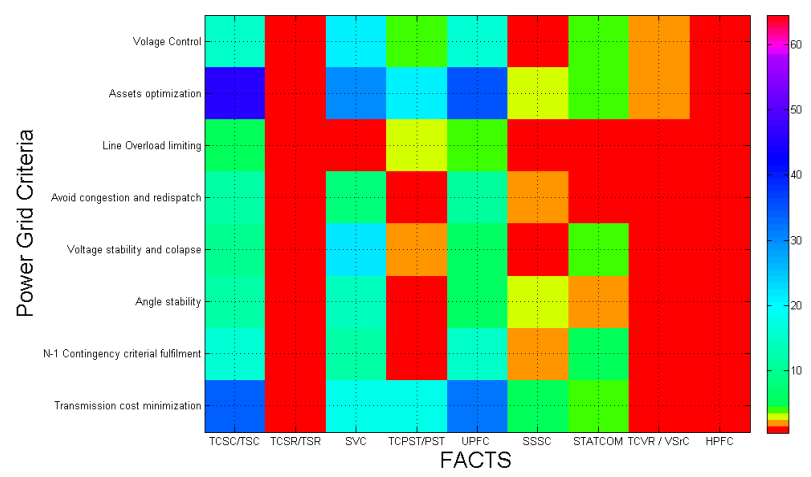

Fig. 5 Cross comparison between FACTS and Grid application

The squares in deep red are the cases where the match has been done less than twice, which means that this FACTS has been lightly studied for this application. The 
squares in blue are the most suitable cases and the ones in green and light blue quite recommended. The most studied cases from the viewpoint of application are:

- Voltage Control: SVC, UPFC, STATCOM, TCSC and TCPST/PST.

- Assets Optimization: SVC, UPFC, STATCOM, TCSC, TCPST/PST and SSSC

- Line Overload Limiting: UPFC, TCSC and TCPST/PST.

- Avoid congestion and re-dispatch: UPFC, TCSC and SVC.

- Voltage stability and collapse: STATCOM, UPFC, TCSC and SVC.

- Angle stability: UPFC, TCSC, SVC and SSSC.

- N-1 Contingency criteria fulfilment: UPFC, TCSC, SVC and STATCOM.

- Transmission cost minimization: UPFC, TCSC, SVC, TCPST/PST, SSSC and STATCOM.

From this comparison and from the studied articles some conclusions can be suggested.

\section{Conclusions}

The data that has been managed in this paper is based in past and recent research papers about FACTS and their applications. The conclusions are obtained from the studied papers and the actual situation of the market. Four different points can be mentioned: application of series and shunt connected devices, type of FACTS, FACTS location and optimization technics and future view of FACTS and their application.

\section{A. Series and shunt FACTS application}

On the one hand, series devices have been used to: optimize assets, minimize transmission cost, reduce line overloads, ensure angle stability and fulfil N-1 contingency criteria. On the other hand, the main objective of shunt connected FACTS has been voltage control and maintenance of stability, assets optimization, avoidance of voltage collapse and transmission cost minimization.

\section{B. Type of FACTS}

The TCSC, SVC and the UPFC are the devices that more deeply have been studied. There are several applications where these FACTS have been used. The TCSC is a wellknown first generation series compensation FACTS whereas the SVC is a parallel compensation device. Those FACTS where introduced in the market many years ago and they have been used world-wide. Nevertheless, a new concept of series and parallel compensation FACTS based on Voltage Source Converters (VSC) has grown up. SSSC and STATCOM, as the new generation FACTS for replacing TCSC and SVC, have been studied for the last years. UPFC, probably one of the most studied FACTS, is basically composed of a SSSC and a STATCOM. UPFC is probably the most flexible FACTS but it is also the most expensive one.

\section{FACTS Location and Optimization Techniques}

From the FACTS optimal location point of view, the methods that have been more deeply researched are GA, Index base sensitivity methods, PSO and classical optimization methods.

Thus, Genetic Algorithm and Particle Swarm Optimization seem to be the two more used methods. Nevertheless, in [26]-[30] a comparison between these and other methods is done. The fact that there is not a dominant technique makes heuristic and meta-heuristics methods unreliable.

Nevertheless, TSOs still trust on continuous power flow studies and sensitivity index based methods to place a FACTS device in the system. Usually, a sensitivity analysis is done based on the Jacobian matrix of the systems. Next, simulation software is used to run historical data of previous cases and to quantify the benefit of the FACTS device in each bus of the system. Normally the result of the power flow obtained is achieved by a quadratic convergent classical method, such as Newton-Raphson.

\section{Future view of FACTS and their application}

UPFC and PST/TCPST are the most flexible FACTS devices but it is necessary to justify their necessity because the required investment for this kind of FACTS is higher than for a single series or parallel type.

An appropriate selection and sizing of FACTS will be the great challenge for the future. The SSSC, as second generation single VSC based FACTS devices, is named to be the future of series FACTS devices. STATCOM will have to deal with the SVC when a parallel FACTS solution is needed. One of the advantages of the STATCOM against the SVC is that it can be easily connected to an energy storage source based on batteries or Ultra-caps. This solution gives the STATCOM the possibility to not only control the voltage but also to inject active power.

\section{Acknowledgement}

The work presented in this paper has been supported by the Basque Government (Ref. IT532-10) and the company Ingeteam Power Technology - Technology.

\section{References}

[1] R, Adapa. "Flexible AC Transmission System (FACTS): System Studies to Assess FACTS Device Requirements on the Entergy System. Electric Power Research Institute. TR-105260. August 1995.

[2] R. Mohan Mathur. "Thyristor based FACTS controllers for electrical transmission systems". IEEE Press Series on Power Engineering, Wiley-Interscience. 2002.

[3] IEEE power \& Energy magazine. "The FACTS on Resolving Transmission Gridlock”. pp.41-46. SeptemberOctober 2003

[4] Yamille E. del Valle. "Optimization of Power System Perfrmance Using FACTS Devices". School of Electrical 
and Computer Engineering. Georgia Institute of Technology. August 2009.

[5] Jordehi, A.R. Jasni, J. "Approaches for FACTS Optimization Problem in Power Systems". Power Engineering and Optimization Conference (PEDCO) Malacca, Malaysia, 2012 IEEE International. 6-7 June 2012

[6] IEEE. "Proposed terms and definitions for flexible AC transmission system (FACTS)". IEEE Transactions on Power Delivery, Vol. 12, No. 4, pp. 1848-1853, October 1997.

[7] Bindeshwar Singh, N. K. Sharma and A. N. Tiwari "A Comprehensive Survey of Optimal Placement and Coordinated Control Techniques of FACTS Controllers in Multi-Machine Power System Environments". Journal of Electrical Engineering \& Technology Vol. 5, No. 1, pp. 79 102, 2010.

[8] Jon Berge, Rajiv. K. Varma, and Wayne H. Litzenberger, "Bibliography of FACTS 2009-2010: Part I, II, III and IV". IEEE Working Group 15.05.17 on HVDC and FACTS Bibliography HVDC and FACTS Sub Committee of IEEE T\&D Committee. 2011.

[9] Mahbube Zaraatzade, Ivana Kockar, and Yong-Hua Song, "Minimizing Balancing Market Congestion Re-dispatch Cost by Optimal Placement of FACTS Devices," Power Tech., 2007, IEEE Lausanne, 1-5 July, 2007 pp.873-878.

[10] Flavio G. M. Lima, Francisco D. Galiana, Ivana Kockar, and Jorge Munoz, "Phase Shifter Placement in Large Scale Systems Via Mixed Integer Linear Programming," IEEE Trans. on Power Systems, Vol. 18, No.3, Aug., 2003.

[11] Tina Orfanogianni, and Rainer Bacher, "Steady-State Optimization in Power Systems with Series FACTS Devices," IEEE Trans on Power Systems, Vol.18, No.1, Feb., 2003.

[12] K. N. Shubhanga, and Anil M. Kulkarni, "Application of Structure Preserving Energy Margin Sensitivity to Determine the Effectiveness of Shunt and Series FACTS Devices," IEEE Trans. on Power Systems,Vol.17, No.3, Aug., 2002.

[13] Seungwon An, John Codren and W.Gedra, "An Ideal Transformer UPFC Model, OPF First-Order Sensitivities, and Application to Screening for Optimal UPFC Locations," IEEE Trans on Power Systems, Vol.22, No.1, Feb., 2007.

[14] Srinivasa Rao Pudi, and S.C.Srivastava, "Optimal Placement of TCSC Based on A Sensitivity Approach for Congestion Management," Fifteenth National Power Systems Conference (NPSC), IIT Bombay, Dec., 2008, pp.558-563.

[15] Y. Mansour, W. Xu. F. Alvarado, and C. Rinzin, "SVC Placement Using Critical Modes of Voltage Stability," IEEE Trans. on Power Systems, Vol.9, pp. 757-762, May, 1994.

[16] S.Gerbex, R. Cherkaoui, and A.J.Germond, "Optimal Location of Multi-Type FACTS Devices in a Power System by Means of Genetic Algorithms," IEEE Trans. on Power Systems, Vol.16, No.3, pp.537-544, Aug., 2001

[17] K. Visakha, D. Thukaram, L. Jenkins, and H.P. Khincha, "Selection of UPFC Suitable Locations for System Security Improvement Under Normal and Network Contingencies," TENCON 2003, Conference on Convergent Technologies for Asia -Pacific Region, Vol-2, 15-17 Oct., 2003, pp.755760.

[18] S.Surender Reddy, M.Sailaja Kumari, M.Sydulu. "Congestion Management in Deregulated Power System by Optimal Choice and Allocation of FACTS Controllers Using Multi-Objective Genetic Algorithm". Transmission and Distribution Conference and Exposition, 2010 IEEE PES.

[19] Sidhartha Panda and Ramnarayan N. Patel," Improving Power System Transient Stability with an Off-centre
Location of Shunt Facts Devices," Journal of Electrical Engineering, VOL. 57, NO. 6, 2006, 365- 368.

[20] Mahdi Karami, Norman Mariun and Mohd Zainal Abdin $\mathrm{Ab}$ Kadir. "On basic definition of optimal allocation of FACTS devices in power system". Proceedings of 2009 IEEE Student Conference on Research and Development (SCORed 2009), 16-18 Nov. 2009, UPM Serdang, Malasia.

[21] Farbod Larki, Homayoun Meshgin Kelk, M. Pishvaei, Ali Johar. "Optimal location of STATCOM and SVC based on contingency voltage stability by using continuation power flow: Case studies of khouzestan power networks in Iran". Second International Conference on Computer and Electrical Engineering. Dubai. 2009

[22] O. L. Bekri, M.K. Fellah. "Optimal location of SVC and TCSC for voltage stability enhancement". The 4th International Power Engineering and Optimization Conference (PEOCO2010), Shah Alam, Selangor, MALAYSIA. 23-24 June 2010

[23] A. Parizad, A. Khazali, M. Kalantar. "Application of HSA and GA in optimal placement of FACTS devices considering voltage stability and losses". International Conference on Electric Power and Energy Conversion Systems, 2009. EPECS '09. Sharjah. 10-12 Nov. 2009

[24] N.M. Tabatabaei, Gh. Aghajani, N.S. Boushehri, S. Shoarinejad. "Optimal location of FACTS devices using adaptive particle swarm optimization hybrid with simulated annealing". International Journal on "Technical and Physical Problems of Engineering" (IJTPE). Published by International Organization on TPE (IOTPE). June 2011.

[25] S. Nagalakshmi, N. Kamaraj. "Secured loadability enhancement with TCSC in transmission system using computational intelligence techniques for pool and hybrid model”. Applied Soft Computing 11 (2011) 4748-4756, Elsevier 17 July 2011.

[26] Yamille del Valle and Ronald G. Harley, Ganesh K. Venayagamoorthy. "Comparison of enhanced-PSO and classical optimization method: A case study for STATCOM placement'. ISAP '09. 15th International Conference on Intelligent System Applications to Power Systems, 2009.

[27] Husam I. Shaheen, Ghamgeen I. Rashed and S. J. Chengz. "Application of differential evolution algorithm for optimal location and parameters setting of UPFC considering power system security". European Transactions on Electrical Power 2009.

[28] H.I. Shaheen, G.I. Rashed, S.J. Cheng. "Application and comparison of computational intelligence techniques for optimal location and parameter setting of UPFC". Engineering Applications of Artificial Intelligence 23 (2010) 203-216, Elsevier.

[29] Masoud Mohammad Rahimi Fard, Heidar Ali Shayanfar, Hossein Shayeghi, Aref Jalili Irani. "UPFC Using For The Congestion Management Lines In Electricity Market Restructured Using PSO and GA Algorithm". Australian Journal of Basic and Applied Sciences, 2011.

[30] D. Mondal, A. Chakrabarti and A. Sengupta. "PSO Based Location and Parameter setting of Advanced SVC Controller with Comparison to GA in Mitigating Small Signal Oscillations". 2011 International Conference on Energy, Automation, and Signal (ICEAS). Bhubaneswar, Odisha. 